\title{
Video Article \\ Dynamic Quantitative Sensory Testing to Characterize Central Pain Processing
}

\author{
Ian G. Mackey ${ }^{1}$, Eric A. Dixon ${ }^{1}$, Kevin Johnson ${ }^{1}$, Jiang-Ti Kong ${ }^{1}$ \\ ${ }^{1}$ Anesthesiology, Perioperative and Pain Medicine, Stanford University School of Medicine
}

Correspondence to: Jiang-Ti Kong at jtkong@stanford.edu

URL: https://www.jove.com/video/54452

DOI: doi: $10.3791 / 54452$

Keywords: Neuroscience, Issue 120, thermal, temporal summation, second pain, contact-heat evoked potential stimulator (CHEPS), conditioned pain modulation, cold pressor test, endogenous analgesia

Date Published: 2/16/2017

Citation: Mackey, I.G., Dixon, E.A., Johnson, K., Kong, J.T. Dynamic Quantitative Sensory Testing to Characterize Central Pain Processing. J. Vis. Exp. (120), e54452, doi:10.3791/54452 (2017).

\section{Abstract}

Central facilitation and modulation of incoming nociceptive signals play an important role in the perception of pain. Disruption in central pain processing is present in many chronic pain conditions and can influence responses to specific therapies. Thus, the ability to precisely describe the state of central pain processing has profound clinical significance in both prognosis and prediction. Because it is not practical to record neuronal firings directly in the human spinal cord, surrogate behavior tests become an important tool to assess the state of central pain processing. Dynamic QST is one such test, and can probe both the ascending facilitation and descending modulation of incoming nociceptive signals via TS and CPM, respectively. Due to the large between-individual variability in the sensitivity to noxious signals, standardized TS and CPM tests may not yield any meaningful data in up to $50 \%$ of the population due to floor or ceiling effects. We present methodologies to individualize TS and CPM so we can capture these measures in a broader range of individuals than previously possible. We have used these methods successfully in several studies at the lab, and data from one ongoing study will be presented to demonstrate feasibility and potential applications of the methods.

\section{Video Link}

The video component of this article can be found at https://www.jove.com/video/54452/

\section{Introduction}

The International Association for the Study of Pain (IASP) defines pain as "an unpleasant sensory and emotional experience." Chronic pain refers to pain that persists beyond 6 months. Chronic pain is a significant problem in the United States, affecting more than 100 million American adults at a cost upwards of $\$ 635$ billion per annum. ${ }^{1}$ Due to the subjective nature of pain, it is difficult for researchers and clinicians to objectively measure a person's painful experience(s), therefore making it challenging to assess and treat pain. For this reason, it is important that we develop standardized tests to quantify pain as objectively as possible. One such test is QST, where standardized sensory stimuli are administered to and rated by the test subject. ${ }^{2}$ There are static and dynamic QST. The former typically assesses the sensory thresholds to or the rating of a single stimulus, while the latter assesses the response to a number of stimuli. ${ }^{3}$ Recently, dynamic QST has gained increasing attention because it offers the opportunity to probe the central processing of incoming nociceptive signals. ${ }^{4,5,6}$

Two key components of dynamic QST are temporal summation (TS) and conditioned pain modulation (CPM). Temporal summation refers to the increased perception of pain from repetitive, noxious stimuli. TS is a behavioral correlate of wind-up, the phenomenon where spinal secondary neurons display increased firing due to repetitive c-fiber input. ${ }^{7,8}$ Generally, TS can be induced by using various noxious stimuli, such as heat, electricity, and tactile methods (i.e. pressure or pinprick), provided that the frequency of the repetitive stimulus is greater than $0.3 \mathrm{~Hz}$, the natural frequency of c-fibers. ${ }^{9,10}$ Many researchers use repetitive heat pulses to generate TS because of the ease in producing and standardizing the noxious heat stimuli. ${ }^{11}$

CPM refers to the phenomenon of "pain inhibits pain," where the presence of a second noxious stimulus decreases the pain perception from an initial noxious stimulus. ${ }^{12}$ The initial noxious stimulus, which is measured before and after (or during) the application of the second stimulus, is referred to as the test stimulus. The test stimulus can be thermal, electrical or tactile. Here thermal stimulus is often used as the testing stimulus because of its ease in adjustment and standardization. ${ }^{13}$ The second stimulus, called the conditioning stimulus, typically consists of a cold or hot water bath applied to a distal extremity. ${ }^{13} \mathrm{CPM}$ is the behavioral correlate of Diffuse Noxious Inhibitory Control (DNIC), a physiological phenomenon where input from peripheral c-fibers results in diffuse inhibition from the brainstem of all incoming stimuli mediated by c-fiber from heterotopic fields. ${ }^{12,14}$

While TS and CPM both have the potential to reflect states and changes in central pain processing, limitations exist in both. ${ }^{4,5}$ For example, because there is a large variability in individuals' sensitivity to heat, the application of a universal stimulus can result in lack of TS in up to $50 \%$ of individuals tested. ${ }^{11,15}$ Similarly, a thermal test stimulus often results in widely different pain ratings that may render CPM test impossible due to floor or ceiling effects. ${ }^{16}$ Therefore, to capture TS and CPM broadly, a protocol that adjusts the heat stimulus to the individual is needed. For 
TS, we adjust the heat pulse temperatures so that they can generate adequate increase in pain rating with each successive pulse; while for the CPM, we adjust the thermal test stimulus to moderately painful (6 out 10) for each individual so that adequate pain ratings may still exist after the application of a conditioning stimulus.

For all psychophysical tests, training of the participant in the proper rating of painful stimuli is crucial to the accuracy and reproducibility of these behavioral tests. ${ }^{17}$ This is particularly relevant for TS when multiple stimuli are presented at a rapid rate and, in the case of CPM, when two different stimuli are applied simultaneously to the participant. Furthermore, for TS from heat pulses, it is especially important to train the participant to rate the c-fiber mediated second pain (slow, burning, usually comes on about 1 second after the heat pulse) and not the first pain (mediated by A-delta fibers and come on immediately with the heat pulse). ${ }^{18,19}$ This is less an issue in CPM as the stimuli there are much longer $(>30 \mathrm{~s})$ and C-fiber mediated sensation would dominate the noxious perception in these situations. ${ }^{19,20}$ In the protocol below, we will go over proper training of participants in detail.

\section{Protocol}

\section{Temporal Summation Protocol}

1. Equipment

1. Heat pulse delivery and location:

1. Used a circular thermode with a diameter of $2.9 \mathrm{~cm}$. This thermode has a microprocessor driven, automated control system, and a peltier element that enables rapid temperature change. Place thermode on the thenar eminence of the subject and use a glove to secure the placement.

2. Computer Interface:

1. Interface the thermode with a software program on a laptop that precisely controls the temperature delivery. Preprogram the appropriate heat stimuli for each stage of the protocol (training 1 and 2 only, as the optimization and final trials follow the same pulse structure as training 2) according to specifications listed in Table 1.

2. Execute the delivery of the heat pulses by selecting the heat stimulus program appropriate for each stage on the software, adjust the temperatures and hit "start" on the user interface.

3. Pain rating device:

1. Use the Computerized Visual Analog Scale (CoVAS).

NOTE: The CoVAS is a box with a mobile lever on a horizontal bar that represents the visual analog scale (VAS). It is an accessory to the main thermode.

2. Training

1. Define the visual analog scale (VAS) on the CoVAS:

1. Present the CoVAS to the participant and define the anchoring points on the VAS: 0 means no pain and 10 means the worst pain imaginable. Ascertain that the participant is comfortable moving the lever left and right with ease.

2. Define second pain:

1. Instruct the participant to focus on second pain from a heat pulse. Define second pain as "a slow, burning, achy pain that takes place about one second after the heat pulse."

3. Rate SECOND pain from a single heat pulse (training trial 1):

1. Secure the thermode on the participant's thenar eminence. Instruct the participant to use CoVAS to rate second pain from each single pulse delivered through the thermode.

2. Begin the pre-programed test on the laptop by hitting the "start" button on the software menu. Each heat pulse lasts 0.5 second and has an inter-stimulus interval of $10 \mathrm{~s}$ (see Fig. 1). Sequentially increase the baseline and peak temperatures of the heat pulse per Table 2.

3. Observe closely the participants' rating of second pain. As soon as the participant rates the second pain as more than $2 / 10$, stop the training trial 1 by pressing the stop button on the software. Remove the thermode and record the final heat pulse parameters (i.e. baseline and peak temperatures).

4. Rate pain continuously from a TS trial (training trial 2):

1. Secure the thermode on the opposite hand. Inform the participant that they will receive a series of 10 rapid pulses. Instruct the participant to rate second pain only, and not focus on the faster heat pain sensation that immediately accompanies each pulse (first pain). Explain to the participant that the second pain may increase, decrease, or remain the same between each pulse.

2. Begin the pre-programmed test on the laptop. Use the final baseline and peak temperatures recorded from step 1.2.3 above. Keep the pulse width at $0.5 \mathrm{~s}$ and the inter-stimulus interval at $2 \mathrm{~s}$ (Fig. 2). Switch hand and repeat this training trial if necessary until the participant is comfortable rating second pain continuously in response to rapid heat pulses.

3. Optimization

1. Define $P 1, P_{\max }$ and $T S_{E}$ :

1. Define $\mathrm{P} 1$ as the pain rating at approximately $2 \mathrm{~s}$ after the delivery of the peak temperature from the first pulse (See Fig. 3). ${ }^{11,21}$ NOTE: $T_{E}$ is the estimated difference between the pain $P 1$ and the maximum pain $\left(P_{\max }\right)$.

2. Adjusting pulse temperature. Aim to achieve $T S_{E}$ between 30 and $70 / 100$ by sequentially adjusting the peak and baseline temperature according to Figure 4. 
NOTE: See Fig. 4 for optimization algorithm.

3. Secure thermode: Using a surgical glove, secure the thermode to the participant's thenar eminence. Switch hand between each trial.

4. Deliver heat pulses: Instruct the participant to only rate their second pain from the heat pulses. Confirm that the participant is ready to begin and then start the pre-programed test on laptop.

5. Iterations: Repeat steps 1.3.2 -1.3.4 until the participant's pain rating at $\mathrm{P} 1$ is $<50 / 100 \mathrm{VAS}$ and their $\mathrm{TS}_{\mathrm{E}}$ is between $30 / 100$ and $70 / 100$ VAS. Conduct no more than 5 optimization trials and ensure that there is a $3 \mathrm{~min}$ rest between each trial. Take the parameters from the very last trial as the final parameters.

4. Final Trials

1. Rest for $5 \mathrm{~min}$ between the optimization trial and the final trials. Repeat 1.3.3 and 1.3.4. using the final parameters from step 1.3 .5 above. Have the participant rest for 3 min and then repeat again.

\section{Conditioned Pain Modulation Protocol}

1. Overview and equipment:

1. Test stimulus: $30 \mathrm{~s}$ contact heat (delivered by the same thermode as above) calibrated to 6 out of 10 pain (Heat-6). See 2.2 for details.

2. Conditioning stimulus:

1. Construct the cold-water bath using a clear plastic box with a perforated dividing wall, and filling it with ice and water on one side and just water on the other. Gently agitate then insert thermometer to water-only side to ensure temperature is stable at $10^{\circ} \mathrm{C}$.

2. If unable to obtain stable temperature, attach a hydro pump to the side with ice to maintain stable temperature by circulating water constantly. Turn on the pump and make sure water side temperature is stable at $10^{\circ} \mathrm{C}$.

3. Timing and computing CPM:

1. Apply the test stimulus to the participant once before and once during the last $30 \mathrm{~s}$ of the conditioning cold bath. Compute CPM as the difference in the pain level of the Heat- 6 stimulus applied before and during the last $30 \mathrm{~s}$ of the cold bath.

2. Calibrating for Heat-6:

1. Estimating Heat- 6 via heat ramp:

1. Secure the thermode to the thenar eminence of the non-dominant hand of the participant. Starting at $32{ }^{\circ} \mathrm{C}$, increase the temperature at a rate of $0.3^{\circ} \mathrm{C}$ per second.

NOTE: A slow ramp rate is used to preferentially activate $\mathrm{C}$-fibers ${ }^{20}$ which are responsible for CPM. ${ }^{12}$

2. Stop whenever the participant reaches thermal tolerance or at $51^{\circ} \mathrm{C}$ maximum. Instruct the participant to rate the pain continuously during the heat ramp. Repeat 3 times and compute the average temperature where the pain rating is $6 / 10$.

2. Fine thresholding: Apply the Heat- 6 and instruct the participant to rate the pain. If the rating is between 5 and $7 / 10$, proceed to 2.3 .2 . If it is $>7$ or $<5$, apply a series of $30 \mathrm{~s}$ thermal heat stimuli to the participant (alternating hands between each stimuli), ranging from $44{ }^{\circ} \mathrm{C}$ to $49^{\circ} \mathrm{C}$ and changing in increments of $0.5-1^{\circ} \mathrm{C}$, until the rating is between $5-7$, allowing a $30 \mathrm{~s}$ rest between each stimulus.

3. Finalizing Heat- 6 and rating of the pre-conditioning, test stimulus:

1. End the fine thresholding once the participant's pain rating is between 5 and $7 / 10$. NOTE: Use this final, within-range pain as the rating of the pre-conditioning testing stimulus for the computation of CPM. Also, record the temperature of the final Heat- 6 that resulted in this rating.

3. CPM protocol

1. Pre-condition test stimulus as per step 2.2.3.

2. Conditioning stimulus:

1. Apply the cold-water bath at $10^{\circ} \mathrm{C}$ to the contralateral foot for $2 \mathrm{~min}$. Instruct the participant to rate the pain from the cold-water bath at 0,30 , and $90 \mathrm{~s}$.

3. Repeating the test stimulus during conditioning stimulus:

1. Apply the final Heat-6 stimulus from fine thresholding (2.2.3) to the same hand again during the last $30 \mathrm{~s}$ of the cold-water bath. Instruct the participant to rate the pain from Heat- 6 at the end of the 30 -second heat stimulus, which is also the end of the $120 \mathrm{~s}$ cold bath.

NOTE: CPM is computed as the difference in the pain rating of the testing stimulus reported before and during the cold bath.

\section{Representative Results}

In an on-going clinical trial where we performed deep phenotyping of patients with chronic axial low back pain, we included dynamic QST as an integral part of the assessment. Table 3 below summarizes baseline data from the first 15 patients where the exact TS and CPM protocol above was used. Note that the data includes patient \#19 because not all 19 recruited patients showed up for their evaluation due to scheduling conflicts and other circumstance. Figure $\mathbf{5}$ visually displays the TS and CPM data side-by-side to reveal patterns of central pain processing changes in these patients. 
As shown above, using an individualized optimization protocol, we obtained TS at the thenar eminence with an average of 2.7 on a $0-10$ VAS scale. We were able to obtain TS in all 19 participants except for Participant 1 , who was not able to reliably identify second pain without being overwhelmed by sensation from the first pain. Participant 13 needed to leave early thus did not undergo the CPM task. Otherwise all 18 participants demonstrated CPM, the average of which is about 3.1. We were successful in achieving some degree of TS and CPM in most of the subjects; and the magnitude of the TS and CPM is consistent with that from the literature. ${ }^{13,15,22}$

Furthermore, as demonstrated in Figure 4, the simultaneous measurement of TS and CPM can lead to insight on a patient's profile in central pain processing. To put it in simplified terms, high TS may indicate abnormally augmented ascending facilitation while a low CPM suggests impaired descending inhibition of nociceptive transmission. For example, Participant 10, showed high TS of 7.7 (out of 10), while Participant 18 demonstrated an essentially absent CPM (-0.5/10). Several pioneer studies have shown that such different profiles of central pain processing may predict different rates of development of chronic pain after surgery, and different responses to drugs that act on specific central pain pathways. ${ }^{4,23,24,25}$ In this example here, it is reasonable to speculate that Participant 10 might respond to Gabapentin, a calcium channel blocker while Participant 18, who has impaired CPM might respond to Duloxetine, a serotonin-norepinephrine reuptake inhibitor. Clearly more data and studies are needed to verify the hypotheses. The interventional trial is ongoing and will assess the response of these back-pain patients to verum and sham electroacupuncture. The individualized QST method presented here would allow us to accurately and longitudinally track the changes in TS and CPM in as many individuals as possible.

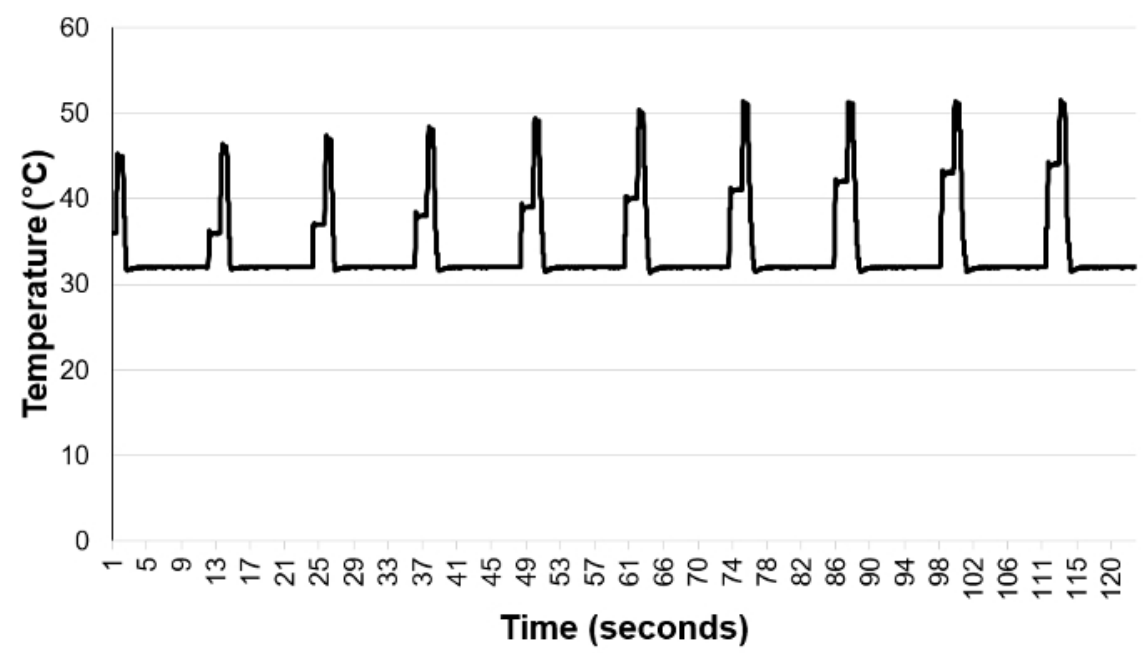

Figure 1. Single Heat Pulses Used in Training Trial 1. Each heat pule lasts $0.5 \mathrm{~s}$ and is $10 \mathrm{~s}$ apart from the next heat pulse. The baseline and peak temperatures of each pulse gradually increase according to Table 2. As soon as the participant perceives second pain from any of the pulses, the endpoint of training trial 1 is reached and the temperature settings of the pulse that leads to perception of second pain are recorded and used in Training Trial 2. Please click here to view a larger version of this figure.

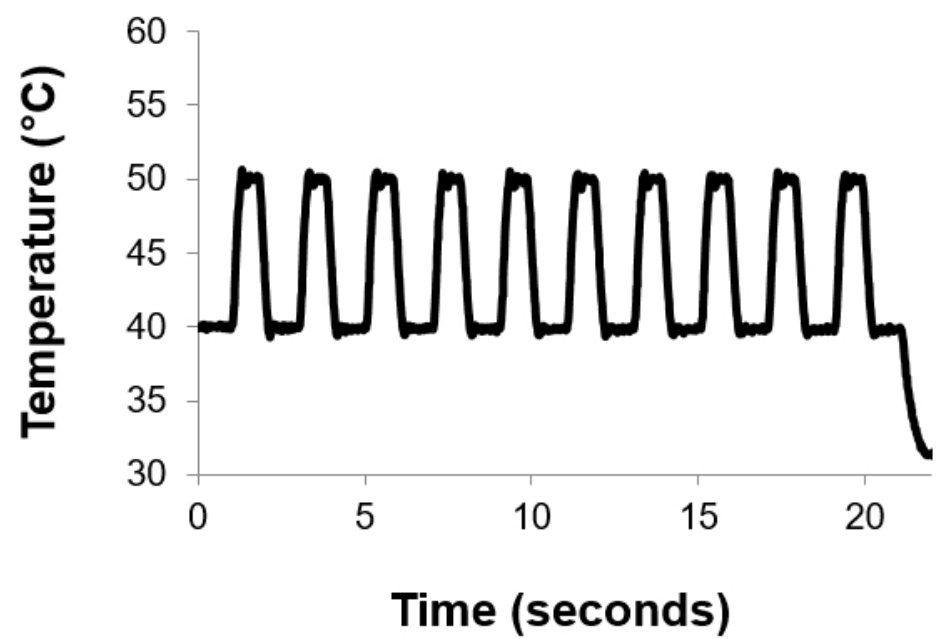

Figure 2. Heat Pulse Train Used in Training Trial 2, Optimization and Final TS Trials. Ten heat pulses, each $0.5 \mathrm{~s}$ long, and $2 \mathrm{~s}$ apart, is delivered in a standard TS trial. 


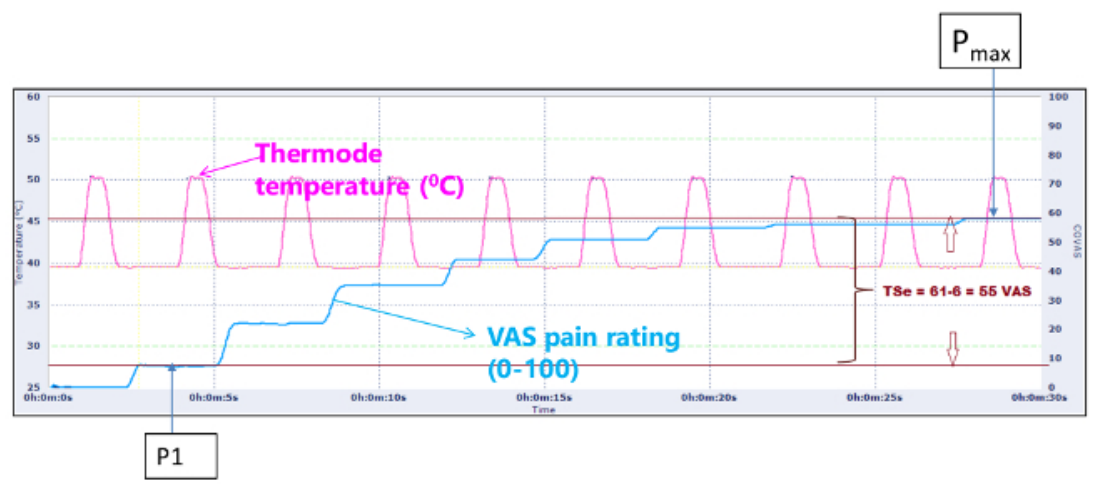

Figure 3. Defining $\mathrm{P} 1, \mathrm{P}_{\max }$ and $\mathrm{TS}_{\mathrm{E}}$. $\mathrm{P} 1$ : rating of the second pain from the first pulse; $\mathrm{P}_{\max }$ : maximum rating of second pain of the entire 10pulse train; $\mathrm{TS}_{\mathrm{E}}$ : estimated magnitude of temporal summation. Please click here to view a larger version of this figure.

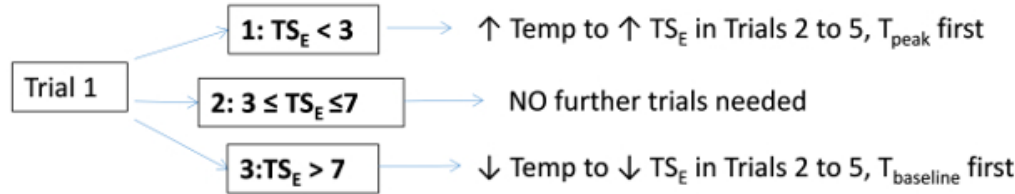

B Scenario 1: If $\mathrm{TS}_{\mathrm{E}}<3$, sequentially increase peak temperature then baseline
temperature by $1^{\circ} \mathrm{C}$ increments in each alternating trial until $\mathrm{TS}_{\mathrm{E}}$ is between 3 and 7
or until 5 optimization trials are completed.

Scenario 2: $\mathrm{TS}_{\mathrm{E}}$ within goal. No further optimization needed.

Scenario 3: $\mathrm{TS}_{\mathrm{E}}>7$, sequentially decrease the baseline temperature and peak temperature by $1^{\circ} \mathrm{C}$ decrements in each alternating trial until $\mathrm{TS}_{\mathrm{E}}$ is between 3 and 7 or until 5 optimization trials are completed.

Figure 4. Algorithms to Individually Optimize Temporal Summation. The objective here is to find baseline and peak pulse temperatures that result in estimated temporal summation (TSE) between 3 and 7 out 10 VAS. If TSE is below goal, increase peak and baseline temperatures by $1{ }^{\circ} \mathrm{C}$ sequentially. If TSE is above goal, decrease baseline and peak temperatures by $1^{\circ} \mathrm{C}$ sequentially. Before the above algorithm, make sure pain rating of the first heat pulse is $\leq 5$ by decreasing the baseline and peak pulse temperatures by small $\left(0.5-1^{\circ} \mathrm{C}\right)$ increments. Please click here to view a larger version of this figure. 


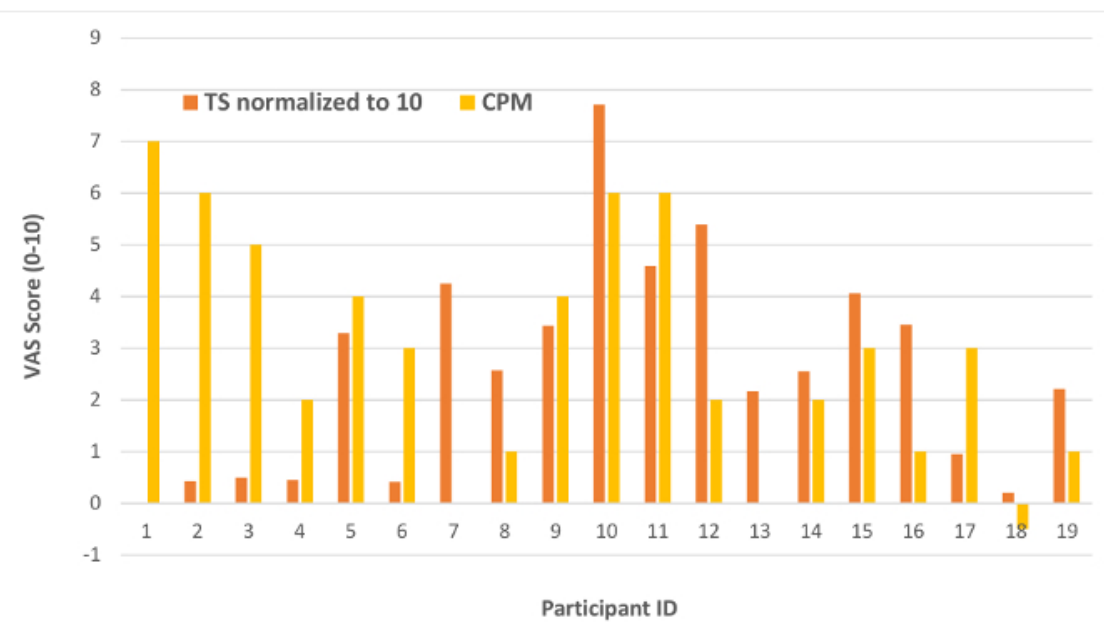

Figure 5. TS and CPM Profiles in Patients from an Ongoing Clinical Trial. Baseline measurement of TS and CPM in the first 19 patients with chronic low back pain from an ongoing clinical trial. The various patterns of relative TS and CPM magnitude reveals potential differences in central pain processing. Please click here to view a larger version of this figure.

\begin{tabular}{|l|l|l|l|l|l|l|}
\hline Trial & \# of pulses & Stimulus Duration & Peak to peak ISI & Ramp Rate & Baseline Temp & Max Temp \\
\hline Training Trial 1 & 1 pulse & $0.5 \mathrm{~s}$ & $10 \mathrm{~s}$ & $40^{\circ} \mathrm{C} / \mathrm{s}$ & $\begin{array}{l}\text { Variable (see Table } \\
\mathbf{2})\end{array}$ & $\begin{array}{l}\text { Variable }(\text { see Table } \\
\mathbf{2}\end{array}$ \\
\hline Training Trial 2 & 10 pulses & $0.5 \mathrm{~s}$ & $2 \mathrm{~s}$ & $40^{\circ} \mathrm{C} / \mathrm{s}$ & $\begin{array}{l}\text { from Training Trial } \\
1\end{array}$ & $\begin{array}{l}\text { from Training Trial } \\
1\end{array}$ \\
\hline Optimization Trials & 10 pulses & $0.5 \mathrm{~s}$ & $2 \mathrm{~s}$ & $40^{\circ} \mathrm{C} / \mathrm{s}$ & $\begin{array}{l}\text { start with temps } \\
\text { from Training Trial } \\
1\end{array}$ & $\begin{array}{l}\text { start with temps } \\
\text { from Training Trial } \\
1\end{array}$ \\
\hline Final Trials & 10 pulses & $0.5 \mathrm{~s}$ & $2 \mathrm{~s}$ & $40^{\circ} \mathrm{C} / \mathrm{s}$ & Individualized & Individualized \\
\hline
\end{tabular}

Table 1: Specifications of Heat Stimuli Used in the TS Protocol (including the training trials).

\begin{tabular}{|l|l|l|}
\hline Pulse Number & Baseline Temperature $\left({ }^{\circ} \mathbf{C}\right)$ & Peak Temperature $\left({ }^{\circ} \mathrm{C}\right)$ \\
\hline 1 & 36 & 45 \\
\hline 2 & 36 & 46 \\
\hline 3 & 37 & 47 \\
\hline 4 & 38 & 48 \\
\hline 5 & 39 & 49 \\
\hline 6 & 40 & 50 \\
\hline 7 & 41 & 51 \\
\hline 8 & 42 & 51 \\
\hline 9 & 43 & 51 \\
\hline 10 & 44 & 51 \\
\hline
\end{tabular}

Table 2: Temperature Settings Used to Capture Second Pain in Training Trial 1. Refer to Figure 1 for shape of single heat pulses delivered with these temperature parameters. 


\begin{tabular}{|l|l|l|l|l|l|}
\hline Subj ID & Base T & Peak T & TS & Heat-6 & CPM \\
\hline & Celsius & Celsius & VAS & Celsius & VAS \\
\hline 1 & & & 0 & 44 & 4.4 \\
\hline 2 & 41 & 51 & 0.43 & 47.6 & 4.717 \\
\hline 3 & 40 & 50 & 0.5 & 44.7 & 4.42 \\
\hline 4 & 38 & 49 & 0.45 & 44 & 4.355 \\
\hline 5 & 42 & 51 & 3.287 & 44 & 4.0713 \\
\hline 6 & 44 & 51 & 0.415 & 45.5 & 4.5085 \\
\hline 7 & 44 & 51 & 4.2495 & 45.5 & 4.12505 \\
\hline 8 & 41 & 51 & 2.575 & 45 & 4.2425 \\
\hline 9 & 44 & 51 & 3.435 & 48 & 4.4565 \\
\hline 10 & 43 & 7.713 & 44 & 3.6287 \\
\hline 11 & 39 & 49 & 4.59 & 42.5 & 3.791 \\
\hline 12 & 39 & 51 & 5.395 & 43.3 & 3.7905 \\
\hline 13 & 44 & 49 & 2.165 & & 0 \\
\hline 14 & 39 & 51 & 2.55 & 44.1 & 4.155 \\
\hline 15 & 40 & 51 & 4.0635 & 44 & 3.99365 \\
\hline 16 & 42 & 51 & 3.45665 & 45.5 & 4.204335 \\
\hline 17 & 40 & 51 & 0.953 & 46.1 & 4.5147 \\
\hline 18 & 37.5 & 2.2135 & 46.5 & 4.529 \\
\hline 19 & 40 & & & 46.63865 \\
\hline
\end{tabular}

Table 3. Results of TS and CPM from an Ongoing Clinical Trial. See Figure 4 for the TS and CPM from the same participants graphed side by side to reveal pain modulation profiles.

\section{Discussion}

\section{Critical Steps within the Protocol}

The TS protocol includes the following in key steps in chronological order: multi-step training (using the visual analog scale to rate pain, rating of second pain from a single heat pulse, and rating second pain from rapid heat pulse trains); optimization of pulse temperatures; obtain TS in 2-3 trials with the optimized temperatures. As with most psychophysical measures, participant training is extremely critical to ensure that pain ratings are consistent across trials and are as accurate as possible. The optimization step is equally important, where both the baseline and peak pulse temperatures are adjusted such that the rating of the first heat pulse is less than 5/10, and the approximated TS is between 3 to 7 .

The key steps of CPM include training of pain rating on visual analog scale, obtaining Heat- 6 from slow heat ramps, confirming Heat- 6 and fine thresholding if necessary, applying a cold bath to contralateral distal extremity and reapplying confirmed Heat- 6 during the last $30 \mathrm{~s}$ of cold bath Similar to the TS protocol, both the training and the individualization of the heat stimulus (Heat-6) are critical in the CPM protocol. Additionally, from experience as well as from the literature, repeating the Heat- 6 stimulus during the last $30 \mathrm{~s}$ of the cold bath is critical and yields a greater magnitude of CPM compared to applying the heat stimulus after the cold bath. ${ }^{26}$ However, given that some individuals cannot tolerate the full 2 min of cold pressor at 10 degree Celsius, it might be reasonable to consider applying the testing stimulus immediately after the completion of the conditioning stimulus to standardize data collection across all individuals.

\section{Modifications and Troubleshooting}

The most common problem with the TS protocol is the inability to obtain TS, which can be due to 3 main causes. First, and most commonly, the pain rating from the first heat pulse may be so strong that it overwhelms the perception of any increase in pain with subsequent pulses (TS). The best way to minimize this problem is to follow the protocol and sequentially decrease the baseline and peak stimulus temperature until the pain rating of the first pulse is less than 5 (out of 10) before optimizing the magnitude of TS. The second cause, opposite to the first one, is when the participant perceives no pain whatsoever at the end of the 10 pulses even at the highest temperature settings. In such situations, one may consider increasing the baseline pulse temperature by 1 or $2{ }^{\circ} \mathrm{C}$. Occasionally, an individual may simply have a hard time discerning and rating second pain, possibly due to both peripheral and central factors. Without reliable perception of second pain, it is very difficult to capture TS. In such situations, we find the best set of the temperatures that an individual can tolerate and record TS as zero.

The most common barriers to a successful CPM protocol are the instability of Heat- 6 and the inability to tolerate a cold bath (10 $\left.{ }^{\circ} \mathrm{C}\right)$ for $2 \mathrm{~min}$. Use the fine thresholding in the current protocol to address the first problem by adjusting heat stimulus temperature step-wise until the pain rating is between 5 and 7 . For the second issue, note that the literature suggests the inhibitory effect from the conditioning stimulus is saturable. ${ }^{27}$ As such, even if a person cannot keep his or her foot in cold bath for $2 \mathrm{~min}$, a sufficient CPM effect should occur with this intensely painful cold stimulus. Modify the protocol to record the duration of the foot submerged in cold water bath and deliver the heat stimulus immediately after the 
participant withdraw his or her foot from the cold bath. CPM is then calculated as the pain rating of the heat stimulus before subtracted by the pain rating of the heat stimulus applied immediately after the cold bath (not during, as the general protocol indicates).

\section{Limitations of Technique}

This method is not without limitations. First, despite our best effort, we were not able to elicit TS and CPM in every individual (missed 1 participant in TS and 1 in CPM, respectively). This, in part, may be due to the large between-individual variability in these parameters. ${ }^{5,15,16,28,29}$ However, the success rate was $94 \%$, which was better than the $50-60 \%$ success rate quoted from the literature. ${ }^{22,28}$ Second, researchers should take caution when interpreting between-individual differences in TS generated by this method since we use different heat pulse temperatures to generate TS in each individual. Therefore, when comparing TS in a cross-sectional sample, one should consider both the differences in the magnitude of TS and in the temperatures used to generate it. The individualized TS method is best suited for longitudinal studies where the focus is on the changes in the same individual overtime. The same concern does not apply to the individualized CPM because the same conditioning stimulus is used for all individuals and only the change in the pain perception of individualized Heat- 6 is recorded and not the raw score of Heat- 6 pain. Although this method allows broad capturing of TS and CPM, it does take more time compared to methods where universal parameters are used. Finally, this technique requires an experienced operator and advanced heat testing machines, both of which are not practical for immediate adaption to busy clinical settings. We encourage future efforts to simplify the methods.

\section{Significance of the Technique with Respect to Existing /Alternative Methods}

Our method of individualizing TS and CPM parameters aim to remove influence of floor and ceiling effect due to variations in peripheral heat sensitivity. The methods presented improved on previous methods published by our group with the goals of both broader capturing and time efficiency. ${ }^{11,30}$ The advantage of individualizing TS and CPM is the ability to capture the state of ascending and descending pain processing in a broad range of individuals, thereby allowing the use of these parameters as a reasonable outcome measure for longitudinal studies.

\section{Future Applications or Directions after Mastering the Technique}

Future studies should focus on additional modifications to save time, collecting of TS and CPM data on large populations to characterize the range of these parameters in individuals who are pain free vs those with chronic pain, and on the correlation of the diversity in the TS and CPM response to specific physiologic processes in addition to windup and DNIC.

\section{Disclosures}

The authors have no conflicts of interests or any financial interests to disclose.

\section{Acknowledgements}

We acknowledge funding support by K23 AT008477 (Kong), NIH K23 DA031808 (Johnson), and the Chris Redlich Endowment in Pain Research (Mackey, Dixon).

\section{References}

1. Pizzo, P. A., \& Clark, N. M. Alleviating suffering 101--pain relief in the United States. N Engl J Med. 366 (3), 197-199 (2012).

2. Backonja, M. M. et al. Value of quantitative sensory testing in neurological and pain disorders: NeuPSIG consensus. Pain. 154 (9), $1807-1819$ (2013).

3. Arendt-Nielsen, L., \& Yarnitsky, D. Experimental and clinical applications of quantitative sensory testing applied to skin, muscles and viscera. J Pain. 10 (6), 556-572 (2009).

4. Cruz-Almeida, Y., \& Fillingim, R. B. Can quantitative sensory testing move us closer to mechanism-based pain management? Pain Med. 15 (1), 61-72 (2014)

5. Curatolo, M., Arendt-Nielsen, L., \& Petersen-Felix, S. Evidence, mechanisms, and clinical implications of central hypersensitivity in chronic pain after whiplash injury. Clin J Pain. 20 (6), 469-476 (2004).

6. Kong, J. T., Schnyer, R. N., Johnson, K. A., \& Mackey, S. Understanding central mechanisms of acupuncture analgesia using dynamic quantitative sensory testing: a review. Evid Based Complement Alternat Med. 2013187182 (2013).

7. Price, D. D. Characteristics of second pain and flexion reflexes indicative of prolonged central summation. Exp. Neurol. 37 (2), $371-387$ (1972).

8. Price, D. D., Hu, J. W., Dubner, R., \& Gracely, R. H. Peripheral suppression of first pain and central summation of second pain evoked by noxious heat pulses. Pain. 3 (1), 57-68 (1977).

9. Arendt-Nielsen, L., \& Petersen-Felix, S. Wind-up and neuroplasticity: is there a correlation to clinical pain? Eur J Anaesthesiol Suppl. 10 1-7 (1995).

10. Eide, P. K. Wind-up and the NMDA receptor complex from a clinical perspective. Eur J Pain. 4 (1), 5-15 (2000).

11. Kong, J. T., Johnson, K. A., Balise, R. R., \& Mackey, S. Test-retest reliability of thermal temporal summation using an individualized protocol. J Pain. 14 (1), 79-88 (2013).

12. Le Bars, D. The whole body receptive field of dorsal horn multireceptive neurones. Brain Res Brain Res Rev. 40 (1-3), 29-44 (2002).

13. Pud, D., Granovsky, Y., \& Yarnitsky, D. The methodology of experimentally induced diffuse noxious inhibitory control (DNIC)-like effect in humans. Pain. 144 (1-2), 16-19 (2009).

14. Yarnitsky, D. et al. Recommendations on terminology and practice of psychophysical DNIC testing. Eur J Pain. 14 (4), 339 (2010).

15. Anderson, R. J. et al. Temporal summation of second pain: Variability in responses to a fixed protocol. Eur J Pain. (2012).

16. Wilson, H., Carvalho, B., Granot, M., \& Landau, R. Temporal stability of conditioned pain modulation in healthy women over four menstrual cycles at the follicular and luteal phases. Pain. 154 (12), 2633-2638 (2013). 
17. Backonja, M. M. et al. Quantitative sensory testing in measurement of neuropathic pain phenomena and other sensory abnormalities. Clin J Pain. 25 (7), 641-647 (2009).

18. Price, D. D., \& Dubner, R. Mechanisms of first and second pain in the peripheral and central nervous systems. J Invest Dermatol. 69 (1), 167-171 (1977).

19. Van Hees, J., \& Gybels, J. C nociceptor activity in human nerve during painful and non painful skin stimulation. J Neurol Neurosurg Psychiatry. 44 (7), 600-607 (1981).

20. Treede, R. D., Meyer, R. A., Raja, S. N., \& Campbell, J. N. Evidence for two different heat transduction mechanisms in nociceptive primary afferents innervating monkey skin. J Physiol. 483 ( Pt 3) 747-758 (1995).

21. Mauderli, A. P., Vierck, C. J., Jr., Cannon, R. L., Rodrigues, A., \& Shen, C. Relationships between skin temperature and temporal summation of heat and cold pain. J Neurophysiol. 90 (1), 100-109 (2003).

22. Granot, M., Granovsky, Y., Sprecher, E., Nir, R. R., \& Yarnitsky, D. Contact heat-evoked temporal summation: tonic versus repetitive-phasic stimulation. Pain. 122 (3), 295-305 (2006).

23. Eisenberg, E., Midbari, A., Haddad, M., \& Pud, D. Predicting the analgesic effect to oxycodone by 'static' and 'dynamic' quantitative sensory testing in healthy subjects. Pain. 151 (1), 104-109 (2010).

24. Granovsky, Y., \& Yarnitsky, D. Personalized pain medicine: the clinical value of psychophysical assessment of pain modulation profile. Rambam Maimonides Med J. 4 (4), e0024 (2013).

25. Yarnitsky, D., Granot, M., Nahman-Averbuch, H., Khamaisi, M., \& Granovsky, Y. Conditioned pain modulation predicts duloxetine efficacy in painful diabetic neuropathy. Pain. 153 (6), 1193-1198 (2012).

26. Wijk, G., \& Veldhuijzen, D. S. Perspective on diffuse noxious inhibitory controls as a model of endogenous pain modulation in clinical pain syndromes. J Pain. 11 (5), 408-419 (2010).

27. Granot, M. et al. Determinants of endogenous analgesia magnitude in a diffuse noxious inhibitory control (DNIC) paradigm: do conditioning stimulus painfulness, gender and personality variables matter? Pain. 136 (1-2), 142-149 (2008).

28. Raphael, K. G., Janal, M. N., Anathan, S., Cook, D. B., \& Staud, R. Temporal summation of heat pain in temporomandibular disorder patients. J Orofac Pain. 23 (1), 54-64 (2009).

29. Robinson, M. E., Bialosky, J. E., Bishop, M. D., Price, D. D., \& George, S. Z. Supra-threshold scaling, temporal summation, and aftersensation: relationships to each other and anxiety/fear. J Pain Res. 3 25-32 (2010).

30. Bernaba, M., Johnson, K. A., Kong, J. T., \& Mackey, S. Conditioned pain modulation is minimally influenced by cognitive evaluation or imagery of the conditioning stimulus. J Pain Res. 7 689-697 (2014). 